\title{
Growth strategy for two-dimensional nanomaterials via a unified model
}

\section{zongkun chen}

University of Konstanz

\section{Ralf Schmid}

University of Konstanz https://orcid.org/0000-0002-9176-270X

\section{Xingkun Wang}

Ocean University of China

\section{Mengqi Fu}

University of Konstanz

\section{Zhongkang Han}

Skolkovo Institute of Science and Technology

\section{Elke Scheer}

University of Konstanz

\section{Minghua Huang}

Ocean University of China

\section{Peter Nielaba}

University of Konstanz

Helmut Coelfen ( $\square$ helmut.coelfen@uni-konstanz.de)

University of Konstanz https://orcid.org/0000-0002-1148-0308

\section{Article}

Keywords: Two-dimensional (2D) materials, wet-chemical precipitation, 2D growth

Posted Date: December 1st, 2021

DOI: https://doi.org/10.21203/rs.3.rs-1079103/v1

License: (c) (i) This work is licensed under a Creative Commons Attribution 4.0 International License.

Read Full License 


\title{
Growth strategy for two-dimensional nanomaterials via a unified model
}

\author{
Zongkun Chen ${ }^{1 \dagger}$, Ralf Schmid ${ }^{1 \xi}$, Xingkun Wang ${ }^{2}$, Mengqi Fu ${ }^{I \S}$, Zhongkang Han ${ }^{3}$, Elke Scheer ${ }^{1 \S}$, Minghua \\ Huang $^{2 *}$, Peter Nielaba ${ }^{1 \xi *}$, Helmut Cölfen ${ }^{1+*}$ \\ ${ }^{1+}$ Department of Chemistry and ${ }^{1 S}$ Department of Physics, University of Konstanz, Universitätsstraße 10, \\ D-78457 Konstanz, Germany \\ ${ }^{2}$ School of Materials Science and Engineering, Ocean University of China, 266100 Qingdao, China \\ ${ }^{3}$ Theory Department, Fritz-Haber-Institut der Max-Planck-Gesellschaft, Berlin, 14195, Germany
}

CorrespondenceEmail: huangminghua@ouc.edu.cn; peter.nielaba@uni-konstanz.de;

helmut.coelfen@uni-konstanz.de.

\begin{abstract}
Two-dimensional (2D) materials prepared by a wet-chemical precipitation route exhibit many unique properties and high potential in various fields. Still, simple, rational and green fabrication of target materials remains challenging due to the lack of a guiding principle. Here, we propose a universal qualitative model for 2D materials grown for layered and non-layered crystal structures by wet-chemical precipitation, which is confirmed by both theoretical simulation and experimental results. It demonstrates that $2 \mathrm{D}$ growth can be obtained by only tuning reaction concentration and temperature. This model not only has been applied to fabricate more than 30 different 2D nanomaterials in water at room temperature in the absence of additives, but also is promising in optimizing the experimental design of numerous other 2D nanomaterials.
\end{abstract}

\section{Main Text}

Two-dimensional (2D) nanomaterials grown by wet-chemical precipitation are an important class of materials, due to their widespread application ${ }^{1-6}$. Since rational preparation is the first and crucial step towards their utilization, understanding their formation process and predicting the growth strategy are becoming progressively more important 7-10. Although comprehensive investigations, including quantitative and empirical ways ${ }^{11-14}$, have been performed, the related models can only be applied to limited crystal systems resulting from the complexity and diversity of different reaction systems ${ }^{7}$. The lack of a reliable and unified model leads to the result that obtaining 2D morphology of target materials depends primarily on an empirical "trial-and-error" method and most synthesis methods vary from one compound to the other. In addition, the existing preparation methods have various technical drawbacks, such as high cost ${ }^{15}$, tedious operation ${ }^{16}$, low product quality ${ }^{17}$, eco-unfriendly conditions and the utilization of surfactants ${ }^{18-20}$. Here, we theoretically propose and experimentally demonstrate that constructing a unified qualitative model is possible and practical to predict the growth strategy and address the aforesaid problems before discovering a widely applicable and satisfactorily quantitative theory. Guided by our model, more than 30 different 2D nanomaterials with layered and non-layered structures have been successfully fabricated in water at room temperature without using additives thus overcoming the previous problems. Besides, this model can guide the synthesis of a wide range of other 2D nanomaterials, and calls for further study of unified qualitative models for directing the preparation of zero- and one-dimensional nanomaterials. 
Forming 2D nanomaterials in solution originates from a special growth habit, which is determined by the relative growth rates of different facets and requires: $R_{2}$ certain facets $\gg R_{\text {other facets }}$ ( $R=$ the actual growth rate $)^{21,22}$. The growth habit is partly dominated by the structure, but could be influenced by growth parameters ${ }^{11}$. Previous studies (Table S1) have shown that concentration and temperature are two common growth parameters ${ }^{13,23}$. Therefore, to obtain a unified model, understanding how growth habits of materials with different crystal structures respond to the change of concentration and temperature is necessary.
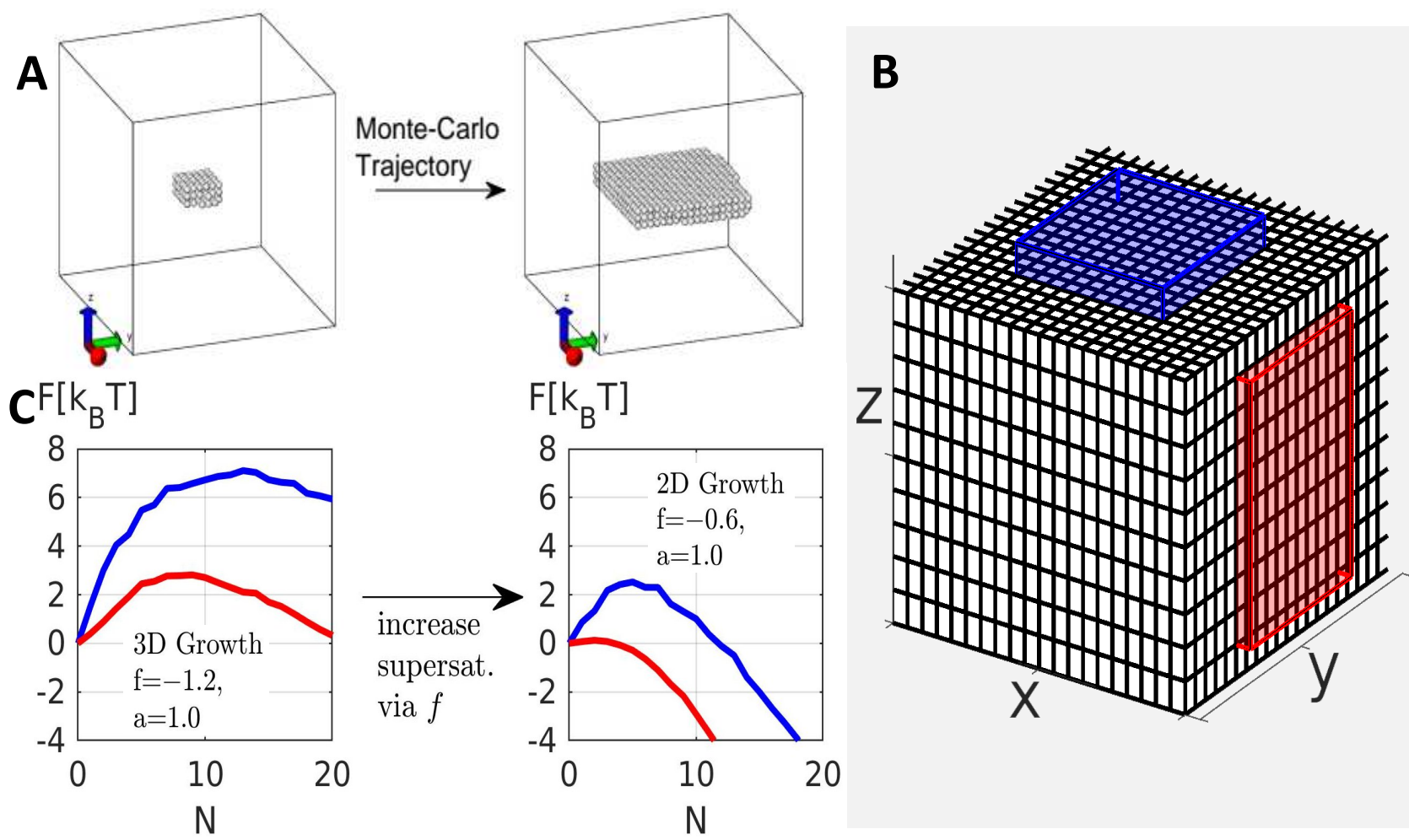

Fig. 1. (A) Snapshots of a Monte Carlo simulation with a cluster seed of size $L_{x y z}=6 \times 6 \times 3, k_{B} T=$ $0.6, a=1.6$ and $f=-1.8$. No thickness increase is observed during growth. (B) Schematic representation of 2D subsystems for simulation of island nucleation on a large flat cluster (red for $x$ and $y$-directional growth, blue for $z$-directional growth). (C) Free Energy barriers of island nucleation in the two subsystems shown in Fig. 1B for two special cases of supersaturation changed by the field $f . N$ is the number of particles in the island. In the case shown here, two different growth scenarios were found for the same value of $a=1$. In one scenario (left), the growth into all directions is bound to a nucleation barrier for smaller supersaturation (i.e. smaller concentration). In contrast, for a larger field $f=-0.6$ (higher concentration), the growth in $z$-direction still suffers from the presence of a nucleation barrier (activated effect) while adding particles in $x$ - and $y$-direction can be performed without costs of energy. In such a case, nanomaterials can grow in a platelet shape as soon as the 3D nucleation barrier is surpassed and the growth speed in $x$ - and $y$ direction is limited by precursor transport in the solution.

To realize this point, a theoretical approach was firstly implemented on an abstract model using basic assumptions for considering the generality. To be specific, Metropolis Monte Carlo (MC) simulations were carried out on the simple cubic lattice using the Ising model (i.e. Q-2-Potts- 
Model) ${ }^{24-26}$. Each lattice point $i$ is assigned the property $m_{i}=1$ and 2 , which represent a crystal particle and a solvent particle, respectively. In the $Q$-2-Potts-Model, the total energy of a given lattice configuration is defined as the sum of next-neighbor couplings of the same occupation $m_{i}$ and an additional field $f$ acting on each lattice point to control supersaturation (and concentration) by scaling the statistical weights of particle types, namely $H=$ $-\sum_{k=1}^{2} \sum_{<i j>} J_{<i j>}(k) \delta\left(m_{i}, k\right) \delta\left(m_{j}, k\right)-f \sum_{i} \delta\left(m_{i}, 1\right)$, where $\delta(\cdot)$ is the Kronecker-Delta and $J_{<i j>}(k)$ represent the anisotropy of the particle interactions. We set $J_{<i j>}(2)=1$ for all directions resembling isotropic interaction between solvent particles, and we set $J_{x}(1)=J_{y}(1)=1+$ $a$ and $J_{z}(1)=1$ for crystal particles. Parameter $a$ is used to describe the anisotropy degree of the interaction energies, reflecting the anisotropic structure difference. During performing $\mathrm{MC}$ simulations, a random site $i$ is chosen and its particle type $\left(m_{i}=1\right.$ or 2$)$ is switched. After calculating the energy difference $\left(\Delta H=H_{\text {new }}-H_{\text {old }}\right)$, the change of lattice configuration is accepted with the probability $p_{a c c}=\min \left[1, \exp \left(-\Delta H /\left(k_{B} T\right)\right)\right]$. For studying the growth habit of materials, the system is initialized in the solvent-dominated state, and the field $f$ is set to values small enough to prevent the systems from suddenly filling with crystal particles.

As the first test on lattice configuration behavior, we simply shoot trajectories with cuboids of different extensions as cluster seeds to check for the presence of nucleation barriers and growth habits. This procedure is done for various values of interaction anisotropy $a \in[0,2] \subset R$ and supersaturation $f \in[-2,1] \subset R$. The temperature is fixed at $k_{B} T=0.6$ so that the equilibrium clusters form flat faces ${ }^{27}$. Note that the simulation runs in the Semi-Grandcanonical Ensemble ${ }^{28}$, i.e. with constant supersaturation. We found that most of the smaller seeds dissolve, and postcritical seeds possess two growth modes, namely 3D growing clusters for smaller values of $a$ and clusters growing in platelet shape for larger values of $a$ (Fig. 1A). This leads to the assumption that there is a parameter space where growth in thickness is an activated process which is known as island nucleation.

To investigate detailly this, we calculated the free energy of island nucleation on an extended cluster with flat faces by cutting out the ad-atom layer and running the simulation procedure only within this 2D subsystem. The fixed large cluster and subsystems (blue and red) are shown schematically in Fig. 1B. For the energy values fed into the MC algorithm, the presence of cluster and solvent is still used, contributing to the result that there is a different situation for the crystal type particles added in the blue subsystem than in the red one since the anisotropy of interactions $a$ impacts on the energy differently. The nucleation barriers calculated this way are shown in Fig. $1 \mathrm{C}$ for some example values of $f$ and $a$, where the color code for $x-y$ and $z$-directional growth was preserved. The barriers for island nucleation are higher for $z$-directional growth than for $x$ - and $y$ directional growth due to $a>0$.

Following the transition state theory ${ }^{29,30}$, the rate of layer by layer growth via island nucleation can be related to the height of the free energy barrier $\Delta F$ by an Arrhenius law $R=v$. $\min \left[1, \exp \left(-\Delta F /\left(k_{B} T\right)\right)\right]$, where $v$ is a constant describing events per time and may strongly depend on diffusion speed, but we set it to one here as the configurational lattice study has no intrinsic time. We stress that for the validity of the transition state theory the barrier needs to be larger than zero and the rates are limited to a maximal value of $v(=1)$ since in the case with no barrier the kinetic pre-factor is dominating the rates. Fig. 2A shows the growth rate differences of the fast growth direction (red subsystem) $R_{f}$ and the slow growth direction (blue subsystem) $R_{s}$ for a mesh of pairs of $f$ and $a$. As the barriers for $R_{f}$ are always smaller or of equal height as for $R_{s}$, the 
resulting profile of rate differences is between zero and one. Regarding the space of $f / a$ values and the corresponding rates, one can find domains representing different growth habits of various materials, as indicated by the red lines in
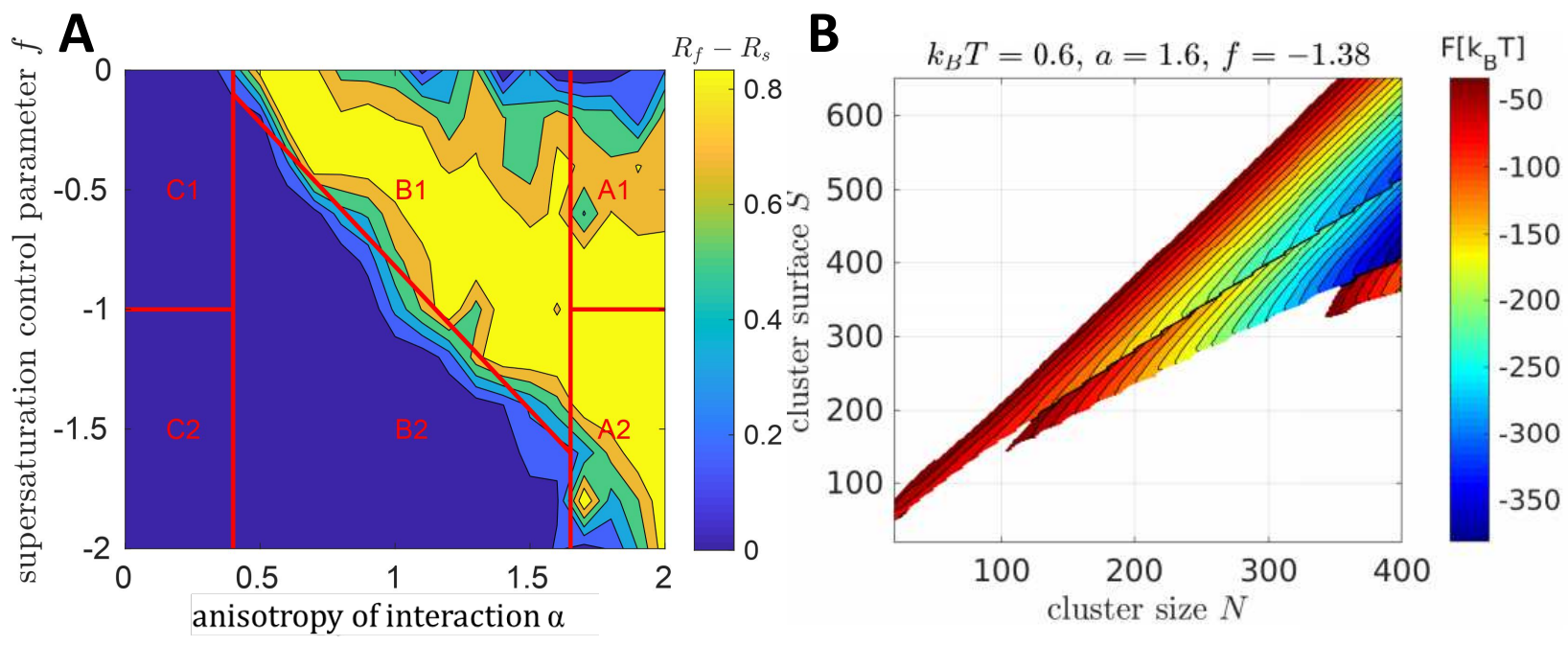

Fig. 2. (A) Difference of growth rates between the slow $z$ direction and the fast $x$ and $y$ directions as proposed by transition state theory for constant kinetic pre-factor $v$. The qualitative alignment of this area in the $(f, a)$-space represents different growth habits of various materials, which is indicated by the dividing marks of material A1-2, B1-2 and C1-2. For small anisotropy $(a)$ in the lattice, the difference in growth rates is small and 3D growth can be observed. If the anisotropy (a) is larger, the growth habit can switch from 2D to 3D growth by changing the supersaturation via $f$. For even larger anisotropy $(a)$, the space of $f$ values resulting in $2 \mathrm{D}$ growth becomes bigger due to the huge difference in growth rates, which indicates the finding that $2 \mathrm{D}$ growth is observed for any concentration for very large anisotropy $(a)$ in the lattice. Note that for very large supersaturations the growth rate difference vanishes even for large anisotropy, which is the case of diffusion limited (i.e. kinetically limited) growth. (B) Nucleation barrier of a nanomaterial growing anisotropically. In this $2 \mathrm{D}$ representation with the free energy dependent on the cluster size $N$ and the cluster surface $S, 2 \mathrm{D}$ growth habit becomes visible by the formation of channels, dividing growth scenarios with different numbers of layers.

Fig. 2A.

Besides, we carried out a 3D simulation of nucleation and calculated the free energy $F(N, S)$ of cluster growth dependent on the nanomaterial size $N$ and its surface $S$. In this Free Energy landscape (Fig. 2B), two separate channels are populated by nanomaterials of different thicknesses. The separating barriers can be identified as island nucleation barriers. The path along the surfaces $N(S)$ corresponding to the minimum Free Energy of each of these channels is given by straight lines, resembling a linear volume to surface dependence due to the $2 \mathrm{D}$ growth. Around a cluster size of $N=350$, a third channel with the next layer shows up. This indicates that for a cluster growing to larger sizes more and more channels become energetically possible but the addition of each layer is still an activated process, confirming again the existence of the island nucleation barrier on the slow growth surface. Note that as diffusion might also influence the growth habits, we also performed finite difference simulations of the concentration profile around a cluster with 
different chemical potentials at the surfaces. The results indicate that the concentration profiles around a cluster seed can differ in the case of smaller vs. larger anisotropy of growth rates, hinting at the possible effect of concentration on the growth habits of materials with different structures. For completeness, similar simulations were implemented on the hexagonal close-packed lattice (hcp) and the same behavior was found. See the supporting information (SI) for additional information about the above analysis.

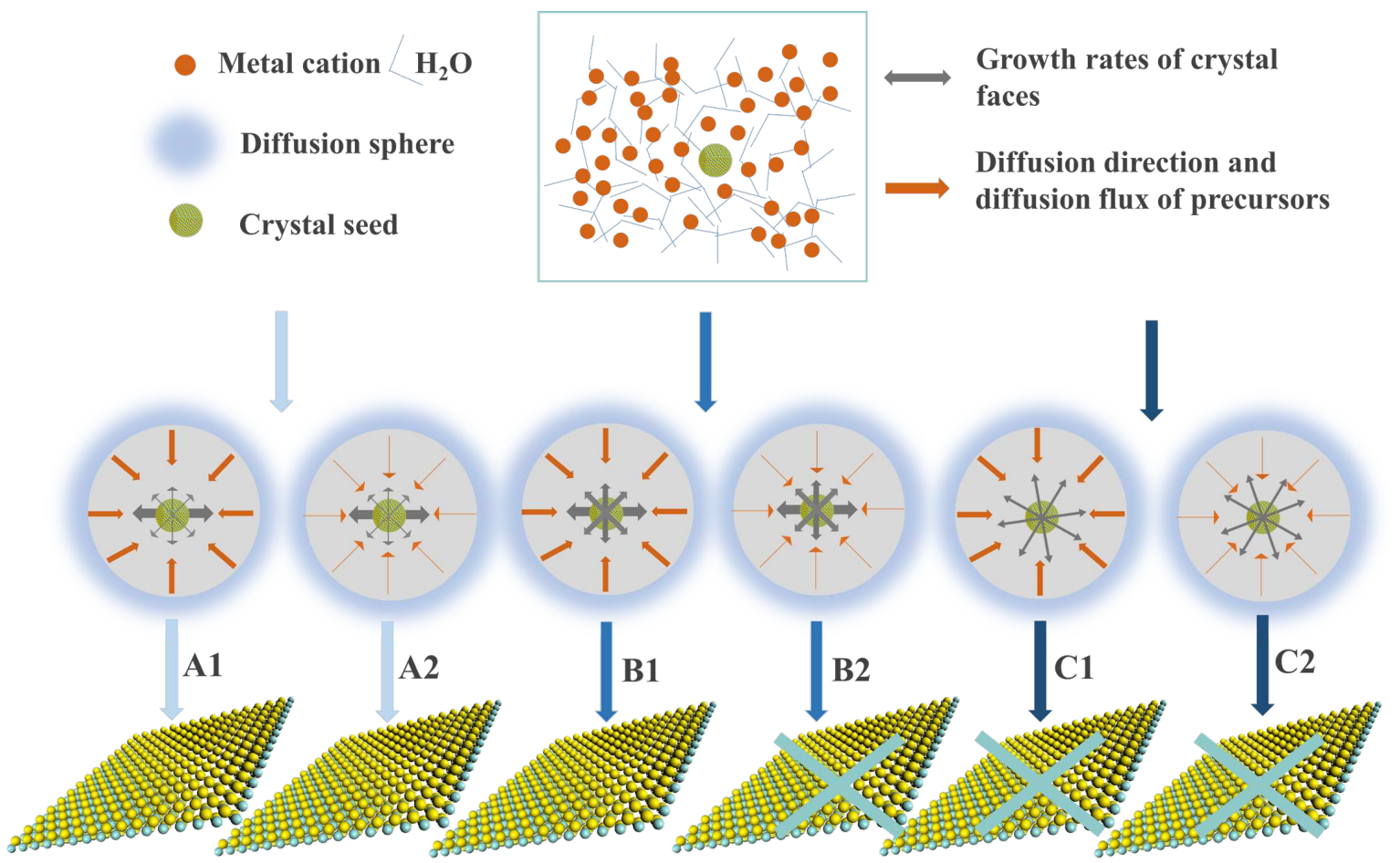

Fig. 3. Schematic representation of the formation mechanism for 2D nanomaterials. Differences in the thickness of the orange and gray arrows represent the symbolized flux amount and the relative growth rate, respectively. Based on the initial growth trend of the seed and the supply of precursors, several cases can be distinguished. (A1) The crystal seed possesses a huge growth rate superiority along one plane at room temperature and enough precursors are supplied by diffusion. 2D nanomaterials are obtained. (A2) differs from (A1) by an insufficient supply of growth precursors. This case also leads to the formation of 2D nanomaterials. (B1) High supply of precursors coupled with medium growth rate differences between different growth directions leads to the formation of 2D nanomaterials. On the contrary, (B2) low supply of precursors coupled with medium growth rate differences brings about a non-2D nanomaterial morphology. (C1 and $\mathrm{C} 2)$ Minor growth rate differences cause forming non-2D morphology no matter if enough or insufficient precursors are supplied at room temperature.

Theoretical simulation results indicate that 2D growth habit depends on the anisotropy degree of the interaction energies, but could be significantly influenced by the change of precursor concentration at a certain temperature. As is known, the crystal growth from solution involves two steps in sequence: ${ }^{31,32}$ the monomers' transport from the bulk solution to the area next to the surface of a crystal seed called diffusion sphere and monomers react with the surface of the crystal. The net transport velocity of monomers is obtained from: 


$$
v=\frac{4 \pi D r\left([C]_{b}-[C]_{S}\right)}{t}
$$

where $D$ is monomers' diffusion constant, $r$ is the seed radius, $t$ is time, $[C]_{b}$ represents the bulk concentration of the solution and $[C]_{S}$ is the monomer concentration at the crystal's surface. Besides, previous studies (Table S1) demonstrated that the reaction rate is related to temperature, concentration, crystal structure, etc. Based on the above analysis, we proposed a possible relationship between 2D growth habit and these two steps influenced by concentration and temperature for materials with different structures. To be specific, for the crystal seed with a preferential growth direction at room temperature, the transport velocity of precursors is the key to maintaining the preferential growth ${ }^{33}$, while it is not necessary to amplify growth rate differences for realizing 2D formation through parameter control (e.g. temperature). Large transport velocity caused by high precursor concentrations guarantees that enough precursors can be supplied for the growth of the fast growth direction and thus maintain the preferential growth (A1 and B1 in Fig. 3). In contrast, the decreased precursor concentration leads to the reduction of transport velocity of precursors, which in turn lowers growth rates of different facets ${ }^{32}$. Such change has a greater impact on the growth rate of preferential direction than other directions, as the preferential growth direction requires more precursors to maintain its speed superiority. Consequently, the superiority of preferential growth could be attenuated (A2) or eliminated (B2) upon the decrease of precursor concentration, and the declined degree is related to the intrinsic growth rate difference between the preferential growth direction and other directions of the crystal seed. For the growth of crystal seed with minor differences along all directions at room temperature, a similar precursor supply is required for these directions ${ }^{34}$. The changed precursor concentration has a comparable effect on growth rates of all directions, inducing the formation of analogous morphologies under varying precursor concentrations. In this case, the lack of preferential growth superiority leads to difficulty in forming 2D morphology $(\mathrm{C} 1$ and $\mathrm{C} 2)$, and attention should be paid to tuning other growth parameters to obtain a preferential growth habit. The growth rate depends exponentially on the temperature ${ }^{13,23}$, and growth rates of different facets have different degrees of response to the change of reaction temperature. Thus, adjusting temperature enables us to amplify the minor growth rate differences and realize $R_{2}$ certain facets $\gg$ $R_{\text {other facets. }}$

To experimentally verify the validity of our model, we conducted a series of wet-chemical precipitation experiments by varying the precursor concentration or reaction temperature but keeping all other parameters constant. More details about synthesis recipes are provided in SI. Fig. S3-1 exhibits schematic representation of set-ups used in our study. Interestingly, 2D morphology of all investigated materials (34 kinds) can be successfully obtained without using additives under certain concentration and temperature. The crystalline phase of these 2D nanomaterials was estimated by X-ray diffraction (XRD). Their XRD patterns (Fig. S3-2) can be well indexed to $\mathrm{Mg}(\mathrm{OH})_{2}, \mathrm{Ni}(\mathrm{OH})_{2}, \mathrm{Ca}(\mathrm{OH})_{2}, \quad \mathrm{Al}(\mathrm{OH})_{3}, \mathrm{Zn}_{5}(\mathrm{OH})_{8} \mathrm{Cl}_{2} \cdot \mathrm{H}_{2} \mathrm{O}, \mathrm{Co}_{1.176}(\mathrm{OH})_{2.004} \mathrm{Cl}_{0.348}\left(\mathrm{H}_{2} \mathrm{O}\right)_{0.454}$, $\mathrm{Cd}_{4} \mathrm{Cl}_{3}(\mathrm{OH})_{5}, \quad \mathrm{Cu}_{2}(\mathrm{OH})_{3}\left(\mathrm{COOCH}_{3}\right) \cdot \mathrm{H}_{2} \mathrm{O}, \quad \mathrm{Eu}(\mathrm{OH})_{\mathrm{x}}\left(\mathrm{NO}_{3}\right)_{3-\mathrm{x}} \cdot \mathrm{nH}_{2} \mathrm{O}, \quad \mathrm{Y}(\mathrm{OH})_{\mathrm{x}}\left(\mathrm{NO}_{3}\right)_{3-\mathrm{x}} \cdot \mathrm{nH}_{2} \mathrm{O}$, $\mathrm{Tb}(\mathrm{OH})_{\mathrm{x}} \mathrm{Cl}_{3-\mathrm{x}} \cdot \mathrm{nH}_{2} \mathrm{O}, \quad \mathrm{Co}\left(\mathrm{S}_{\mathrm{x}} \mathrm{OH}_{\mathrm{y}}\right), \quad \mathrm{Mn}_{3} \mathrm{O}_{4}, \quad \gamma-\mathrm{Fe}_{2} \mathrm{O}_{3}, \quad \mathrm{CeO}_{2}, \quad \mathrm{~Pb}_{3} \mathrm{O}_{2}(\mathrm{OH})_{2}, \quad \mathrm{CuS}, \mathrm{H}_{2} \mathrm{WO}_{4}$, $\left(\mathrm{NH}_{4}\right)_{2} \mathrm{Mo}_{3} \mathrm{~S}_{13}, \mathrm{Mn}_{3}\left(\mathrm{PO}_{4}\right)_{2} \cdot 3 \mathrm{H}_{2} \mathrm{O}, \mathrm{Cr}(\mathrm{OH})_{3}, \mathrm{Sb}_{2} \mathrm{O}_{3}, \mathrm{CrOCl}, \mathrm{Fe}$ doped $\mathrm{Co}(\mathrm{OH})_{2}, \mathrm{Cu}_{4} \mathrm{SO}_{4}(\mathrm{OH})_{6}$, $\mathrm{ZnO}, \mathrm{Co}(\mathrm{OH})_{2}, \mathrm{FeOOH}, \mathrm{Bi}(\mathrm{OH})_{3}, \mathrm{In}_{2} \mathrm{~S}_{3}, \mathrm{BaSO}_{4}, \mathrm{FePO}_{4}, \mathrm{Co}_{3}\left(\mathrm{PO}_{4}\right)_{2} \cdot \mathrm{nH}_{2} \mathrm{O}$ and $\mathrm{Fe}$ doped $\mathrm{Ni}(\mathrm{OH})_{2}$ phases, respectively. The involved elements in our study cover most of the metallic groups in the periodic table (Fig. S3-3), suggesting that our model is promising for guiding the unified fabrication of 2D nanomaterials. Energy dispersive X-ray (EDX) spectroscopy and Fourier 
transform infrared (FTIR) spectroscopy (Fig. S3-4) were utilized to verify the existence of intercalated anions in hydroxides with intercalated anions. Typical 2D nanosheet or nanoplate morphology can be easily identified from scanning electron microscopy (SEM, Fig. S3-5) and transmission electron microscopy (TEM, Fig. 4) images. To determine the exact thickness of these 2D nanomaterials, atomic force microscopy (AFM) was utilized. The obtained results indicate that most 2D nanomaterials possess a thickness less than $5 \mathrm{~nm}$. The thicknesses of $\mathrm{Mg}(\mathrm{OH})_{2}$, $\mathrm{Cu}_{2}(\mathrm{OH})_{3}\left(\mathrm{COOCH}_{3}\right) \cdot \mathrm{H}_{2} \mathrm{O}$ and $\mathrm{Mn}_{3} \mathrm{O}_{4}$ nanomaterials, consisting of many thin layers as observed in Fig. S3-5-1A, 3-5-1H and 3-5-2E, seem to be larger than $10 \mathrm{~nm}$ (Fig. S3-6).

To verify whether our experimental results are in accord with the predicted routes, it is necessary to explore the morphology response to the corresponding reaction parameters (concentration and temperature) for all reaction conditions. The investigated materials can be classified into three cases according to how the morphology responds to the changed reaction temperature or concentration. In the first case, the experimental results (Fig. S3-7-1) demonstrate that some materials, namely $\mathrm{Ni}(\mathrm{OH})_{2}, \mathrm{Mg}(\mathrm{OH})_{2}, \mathrm{Ca}(\mathrm{OH})_{2}, \mathrm{Co}_{1.176}(\mathrm{OH})_{2.004} \mathrm{Cl}_{0.348}\left(\mathrm{H}_{2} \mathrm{O}\right)_{0.454}, \mathrm{Eu}(\mathrm{OH})_{x}\left(\mathrm{NO}_{3}\right)_{3}$ $\mathrm{x} \cdot \mathrm{nH}_{2} \mathrm{O}, \mathrm{Y}(\mathrm{OH})_{\mathrm{x}}\left(\mathrm{NO}_{3}\right)_{3-\mathrm{x}} \cdot \mathrm{nH}_{2} \mathrm{O}, \gamma-\mathrm{Fe}_{2} \mathrm{O}_{3}$ and $\mathrm{Pb}_{3} \mathrm{O}_{2}(\mathrm{OH})_{2}$, always tend to form 2D morphology under the reaction condition of varied concentration at constant temperature or changed temperature at a certain concentration. This phenomenon indicates that the preferential growth habit along one direction, to some degree, cannot be influenced by the changed concentration or temperature, agreeing well with the predicted route A in our model. Note that these mentioned materials have layered structure (Fig. S3-8). Such anisotropic crystal structure contributes to the massive formation energy difference of different facets, which leads to the huge growth rate difference between the growth along in-plane direction and other directions, thus enabling nanomaterials to preferentially grow along the in-plane direction. Selected area electron diffraction (SAED) and high-resolution TEM were utilized to investigate the exposed facets for verifying such growth habit of the obtained 2D nanomaterials. The results (Fig. S3-8) confirm their preferential growth along the in-plane direction.

In the second case, for $\mathrm{Zn}_{5}(\mathrm{OH})_{8} \mathrm{Cl}_{2} \cdot \mathrm{H}_{2} \mathrm{O}, \mathrm{Al}(\mathrm{OH})_{3}, \mathrm{Cd}_{4} \mathrm{Cl}_{3}(\mathrm{OH})_{5}, \mathrm{Cu}_{2}(\mathrm{OH})_{3}\left(\mathrm{COOCH}_{3}\right) \cdot \mathrm{H}_{2} \mathrm{O}$, $\mathrm{Tb}(\mathrm{OH})_{\mathrm{x}} \mathrm{Cl}_{3-\mathrm{x}} \cdot \mathrm{nH}_{2} \mathrm{O}, \mathrm{CuS}, \mathrm{H}_{2} \mathrm{WO}_{4}$ and $\mathrm{CrOCl}$ materials, the experimental results (Fig. S3-7-2) demonstrate that different reaction temperatures invariably promotes the formation of $2 \mathrm{D}$ morphology, while higher and lower precursor concentrations induce the generation of 2D and non-2D morphology, respectively. These results indicate that their final morphologies may not be influenced by varying the reaction temperature, but are directly associated with precursor concentrations. This is in line with the predicted route B in our model. Since these materials possess layered structure (Fig. S3-8), as mentioned in the first case, such materials tend to preferentially grow along the in-plane direction and thus have the same response as the first case on high precursor concentration and the change of the reaction temperature. Besides, the formation of non-2D morphology at low precursor concentration can be attributed to the fact that the change of precursor concentration has a huge impact on the preferential growth superiority of these materials. To be specific, low precursor concentration can result in the decreased transport velocity of precursors, which can in turn eliminate the preferential growth superiority and thus facilitates the formation of non-2D morphology. Both materials of route A and route B possess layered structure and preferential growth habit along in-plane direction. However, different responses of their growth habits to the decreased precursor concentration originate from their different degree of growth rate difference, which can be reflected in the surface energy difference of different directions. Taking $\mathrm{Ni}(\mathrm{OH})_{2}$ and $\mathrm{Zn}_{5}(\mathrm{OH})_{8} \mathrm{Cl}_{2} \cdot \mathrm{H}_{2} \mathrm{O}$ as examples (Table S2), the former has a 
smaller surface energy for (001) plane than for other planes, while the latter's surface energy for
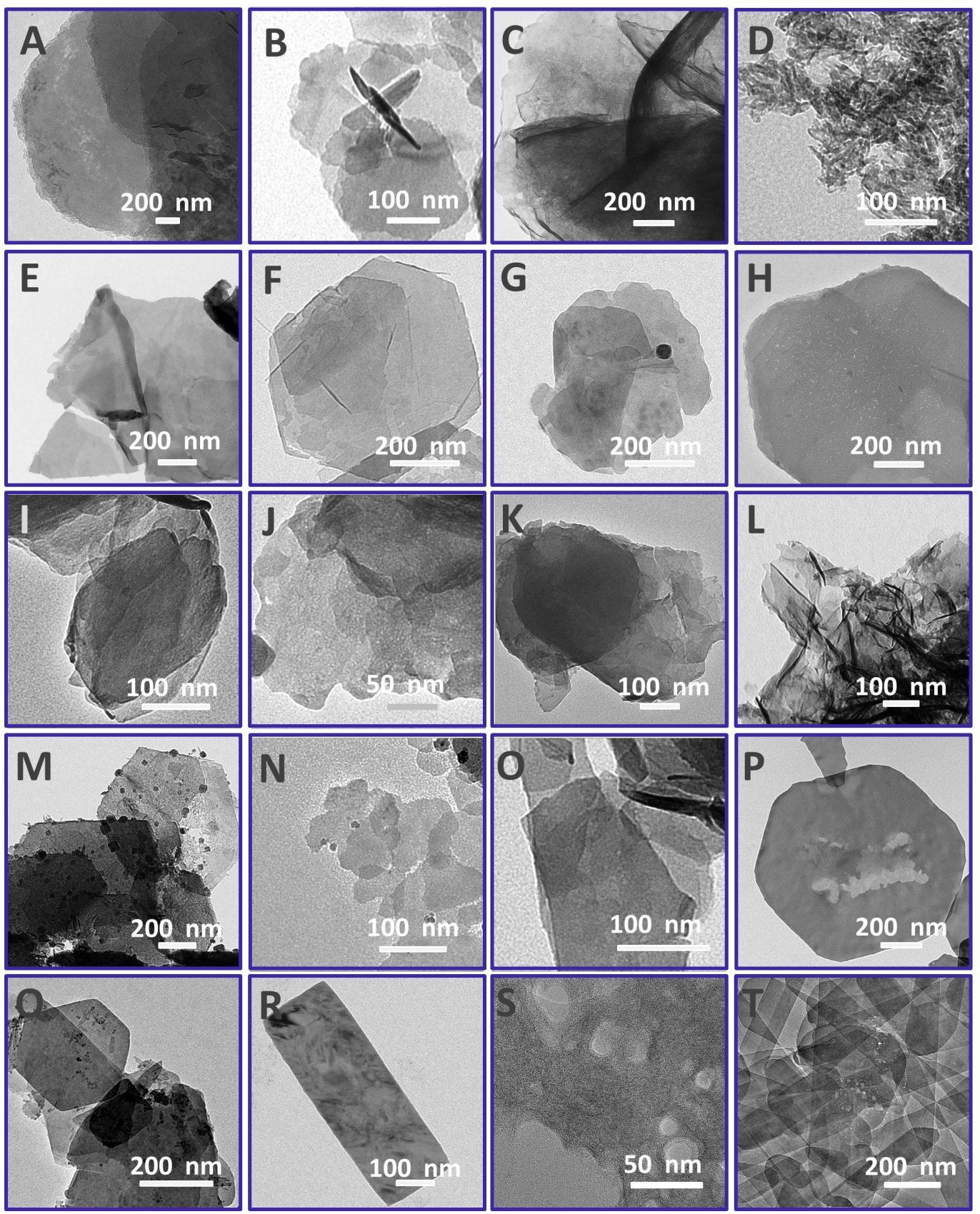

Fig. 4. TEM images of $2 \mathrm{D}$ nanomaterials. (A) $\mathrm{Mg}(\mathrm{OH})_{2}$, (B) $\mathrm{Ni}(\mathrm{OH})_{2},(\mathrm{C}) \mathrm{Ca}(\mathrm{OH})_{2}$, (D) $\mathrm{Al}(\mathrm{OH})_{3}$, (E) $\quad \mathrm{Zn}_{5}(\mathrm{OH})_{8} \mathrm{Cl}_{2} \cdot \mathrm{H}_{2} \mathrm{O}, \quad$ (F) $\quad \mathrm{Co}_{1.176}(\mathrm{OH})_{2.004} \mathrm{Cl}_{0.348}\left(\mathrm{H}_{2} \mathrm{O}\right)_{0.454}, \quad$ (G) $\mathrm{Cd}_{4} \mathrm{Cl}_{3}(\mathrm{OH})_{5}, \quad$ (H) $\mathrm{Cu}_{2}(\mathrm{OH})_{3}\left(\mathrm{COOCH}_{3}\right) \cdot \mathrm{H}_{2} \mathrm{O}, \quad$ (I) $\quad \mathrm{Eu}(\mathrm{OH})_{\mathrm{x}}\left(\mathrm{NO}_{3}\right)_{3-\mathrm{x}} \cdot \mathrm{nH}_{2} \mathrm{O}, \quad$ (J) $\quad \mathrm{Y}(\mathrm{OH})_{\mathrm{x}}\left(\mathrm{NO}_{3}\right)_{3-\mathrm{x}} \cdot \mathrm{nH}_{2} \mathrm{O}, \quad(\mathrm{K})$ $\mathrm{Tb}(\mathrm{OH})_{\mathrm{x}} \mathrm{Cl}_{3-\mathrm{x}} \cdot \mathrm{nH}_{2} \mathrm{O}$, (L) $\mathrm{Co}\left(\mathrm{S}_{\mathrm{x}} \mathrm{OH}_{\mathrm{y}}\right),(\mathrm{M}) \mathrm{Mn}_{3} \mathrm{O}_{4}$, (N) $\gamma-\mathrm{Fe}_{2} \mathrm{O}_{3},(\mathrm{O}) \mathrm{CeO}_{2}$, (P) $\mathrm{Pb}_{3} \mathrm{O}_{2}(\mathrm{OH})$ 2. (Q) $\mathrm{CuS},(\mathrm{R}) \mathrm{H}_{2} \mathrm{WO}_{4}$, (S) $\left(\mathrm{NH}_{4}\right)_{2} \mathrm{Mo}_{3} \mathrm{~S}_{13}$, and $(\mathrm{T}) \mathrm{Mn}_{3}\left(\mathrm{PO}_{4}\right)_{2} \cdot 3 \mathrm{H}_{2} \mathrm{O}$.

(001) plane is comparable to that of other planes.

In the third case, various experiments by changing precursor concentration have been carried out at room temperature for some materials $\left(\mathrm{Cr}(\mathrm{OH})_{3}, \mathrm{Bi}(\mathrm{OH})_{3}, \mathrm{In}_{2} \mathrm{~S}_{3}\right.$ and $\left.\mathrm{FePO}_{4}\right)$, and the obtained nanomaterials invariably possess similar non-2D morphology (Fig. S3-7-3), indicating that their 
growth habit cannot be impacted by the varied precursor concentration at room temperature. Note that these materials possess non-layered structure (Fig. S3-8) with chemical bonds in all dimensions, meaning that their different facets possess similar surface energy and minor growth rate differences. Since the similar growth rate leads to the comparable requirement for the precursor supply, the change of transport velocity caused by the varied precursor concentration has an analogous effect on influencing growth rates of different facets, leading to the tendency of forming non-2D morphology at a certain temperature. Interestingly, nanoplate morphologies (Fig. S3-7-3) are obtained via tuning the synthesis temperature to 4 or $95^{\circ} \mathrm{C}$, indicating the dependence of morphology on the reaction temperature for these non-layered materials, experimentally supporting the rationality of the mentioned means in route $C$. Detailed comparisons about the predicted routes and experimental results are listed in Table S3, and their consistency can be identified easily, verifying the validity of our model in guiding the synthesis of 2D nanomaterials. As this model is dependent on tuning reaction concentration or temperature on the basis of the intrinsic structure of target materials, it is expected that a variety of other potential 2D materials can be synthesized under the guidance of this model. For other materials with layered unit cells (Fig. S3-9), the formation of 2D morphology should be possible at room temperature via tuning precursor concentration as manifested in the aforementioned routes $\mathrm{A}$ and $\mathrm{B}$. For other materials with non-layered crystal structures, inspired by route $\mathrm{C}$, their $2 \mathrm{D}$ morphology may be obtained via altering the reaction temperature.

Except for the wide applicability, our study possesses several other important advantages. These are

1) the obtained 2D nanomaterials can be transformed into other $2 \mathrm{D}$ nanomaterials via the topochemical transformation approach, further widening the range of synthesizable 2D nanomaterials in our study. For example, $\left(\mathrm{NH}_{4}\right)_{2} \mathrm{Mo}_{3} \mathrm{~S}_{13}, \mathrm{Co}_{1.176}(\mathrm{OH})_{2.004} \mathrm{Cl}_{0.348}\left(\mathrm{H}_{2} \mathrm{O}\right)_{0.454}$ and $\mathrm{Ni}(\mathrm{OH})_{2}$ nanosheets have been converted into $\mathrm{MoS}_{2}, \mathrm{Co}_{3} \mathrm{O}_{4}$ and $\mathrm{NiO}$ nanosheets, respectively, via heat treatment (Fig. S3-10);

2) it is easy to scale up to obtain 1 gram or even 30 grams for some 2D nanomaterials (Fig. S3$11)$;

\section{CONCLUSIONS}

In conclusion, a unified qualitative model of predicting growth strategy for 2D nanomaterials grown by wet-chemical precipitation has been established, and its rationality is confirmed by both theoretical simulation and experimental results. The key point consists of obtaining a preferential growth along two crystal axes spanning a 2D area, which is realized via tuning external growth parameters based on the intrinsic structure of target materials. Under the guidance of our model, 34 chemically different 2D nanomaterials, including oxides, hydroxides, acids, sulfides, oxychlorides and phosphates, have been successfully fabricated, and the synthesis process possesses attractive advantages, including the wide applicability, low production cost, simple operation and upscaling possibility to the gram range. Besides, we believe this model can guide us to improve the synthesis condition of a wide range of other 2D nanomaterials.

\section{Data availability}


The data supporting the findings of this study are available from the corresponding authors upon reasonable request.

\section{Code availability}

The codes used for the findings of this study and README files are freely available for download at https://cloud.uni-konstanz.de/index.php/s/ZDdA67MbsJqRmyY. The included README files describe the detailed information for running smoothly our codes, including the required software and the installation guide, the programs covered by our codes, the instructions for operation performed by our codes, etc.

\section{References}

1. Mounet, N. et al. Two-dimensional materials from high-throughput computational exfoliation of experimentally known compounds. Nature nanotechnology 13, 246-252 (2018).

2. Yin, H. \& Tang, Z. Ultrathin two-dimensional layered metal hydroxides: an emerging platform for advanced catalysis, energy conversion and storage. Chemical Society reviews 45, 4873-4891 (2016).

3. Yan, Z. et al. Anion insertion enhanced electrodeposition of robust metal hydroxide/oxide electrodes for oxygen evolution. Nature communications 9, 2373 (2018).

4. $\mathrm{Hu}, \mathrm{Z}$. et al. Rapid mass production of two-dimensional metal oxides and hydroxides via the molten salts method. Nature communications 8, 15630 (2017).

5. Wang, H.F., Tang, C., Wang, B., Li, B.Q. \& Zhang, Q. Bifunctional Transition Metal Hydroxysulfides: Room-Temperature Sulfurization and Their Applications in Zn-Air Batteries. Advanced Materials 29, 1702327 (2017).

6. Long, X. et al. Metallic iron-nickel sulfide ultrathin nanosheets as a highly active electrocatalyst for hydrogen evolution reaction in acidic media. Journal of the American Chemical Society 137, 11900-11903 (2015).

7. Anderson, M.W. et al. Predicting crystal growth via a unified kinetic three-dimensional partition model. Nature 544, 456-459 (2017).

8. Auyeung, E. et al. DNA-mediated nanoparticle crystallization into Wulff polyhedra. Nature 505, 73-77 (2014).

9. Liao, H.G. et al. Facet development during platinum nanocube growth. Science 345, 916919 (2014).

10. Chung, J. et al. Molecular modifiers reveal a mechanism of pathological crystal growth inhibition. Nature 536, 446-450 (2016).

11. Liu, X.-Y. \& Bennema, P. Morphology of crystals: Internal and external controlling factors. Physical Review B 49, 765-775 (1994).

12. Lovette, M.A. et al. Crystal shape engineering. Industrial \& engineering chemistry research 47, 9812-9833 (2008).

13. Liu, X.Y., Boek, E.S., Briels, W.J. \& Bennema, P. Prediction of crystal growth morphology based on structural analysis of the solid-fluid interface. Nature 374, 342-345 (1995).

14. Lupulescu, A.I. \& Rimer, J.D. In situ imaging of silicalite-1 surface growth reveals the mechanism of crystallization. Science 344, 729-732 (2014). 
15. Zhao, H. et al. A Generalized Strategy for the Synthesis of Large-Size Ultrathin TwoDimensional Metal Oxide Nanosheets. Angewandte Chemie International Edition 56, 8766-8770 (2017).

16. Zhao, C., Zhang, H., Si, W. \& Wu, H. Mass production of two-dimensional oxides by rapid heating of hydrous chlorides. Nature communications 7, 12543 (2016).

17. Liu, X., Ma, R., Bando, Y. \& Sasaki, T. Layered cobalt hydroxide nanocones: microwaveassisted synthesis, exfoliation, and structural modification. Angewandte Chemie International Edition 49, 8253-8256 (2010).

18. Xu, J., Chen, X., Xu, Y., Du, Y. \& Yan, C. Ultrathin 2D Rare - Earth Nanomaterials: Compositions, Syntheses, and Applications. Advanced Materials, 1806461 (2019).

19. Cheng, H., Kamegawa, T., Mori, K. \& Yamashita, H. Surfactant-Free Nonaqueous Synthesis of Plasmonic Molybdenum Oxide Nanosheets with Enhanced Catalytic Activity for Hydrogen Generation from Ammonia Borane under Visible Light. Angewandte Chemie International Edition 53, 2910-2914 (2014).

20. Sun, Z. et al. Generalized self-assembly of scalable two-dimensional transition metal oxide nanosheets. Nature communications 5, 3813 (2014).

21. Mullin, J.W. Crystallization. (Elsevier, 2001).

22. Polarz, S. Shape matters: anisotropy of the morphology of inorganic colloidal particlessynthesis and function. Advanced Functional Materials 21, 3214-3230 (2011).

23. Liu, H. et al. Self-assembly of octachloroperylene diimide into 1D rods and 2D plates by manipulating the growth kinetics for waveguide applications. Chemical communications 50, 4620-4623 (2014).

24. Metropolis, N., Rosenbluth, A.W., Rosenbluth, M.N., Teller, A.H. \& Teller, E. Equation of state calculations by fast computing machines. The journal of chemical physics 21, 1087$1092(1953)$.

25. Landau, D.P. \& Binder, K. A guide to Monte Carlo simulations in statistical physics. (Cambridge university press, 2014).

26. Pan, A.C. \& Chandler, D. Dynamics of nucleation in the Ising model. The Journal of Physical Chemistry B 108, 19681-19686 (2004).

27. Bikker, R., Barkema, G. \& Van Beijeren, H. Equilibrium crystal shapes in the Potts model. Physical Review E 64, 016125 (2001).

28. Duff, N. \& Peters, B. Nucleation in a Potts lattice gas model of crystallization from solution. The Journal of chemical physics 131, 184101 (2009).

29. Laidler, K.J. \& King, M.C. Development of transition-state theory. The Journal of physical chemistry 87, 2657-2664 (1983).

30. Vanden-Eijnden, E. \& Tal, F.A. Transition state theory: Variational formulation, dynamical corrections, and error estimates. The Journal of chemical physics 123, 184103 (2005).

31. Thanh, N.T., Maclean, N. \& Mahiddine, S. Mechanisms of nucleation and growth of nanoparticles in solution. Chemical reviews 114, 7610-7630 (2014).

32. Peng, Z.A. \& Peng, X. Mechanisms of the shape evolution of CdSe nanocrystals. Journal of the American Chemical Society 123, 1389-1395 (2001).

33. Hu, J.Q. et al. A simple and effective route for the synthesis of crystalline silver nanorods and nanowires. Advanced Functional Materials 14, 183-189 (2004).

34. Wang, F. et al. Two-Dimensional Non-Layered Materials: Synthesis, Properties and Applications. Advanced Functional Materials 27, 1603254 (2017). 


\section{Acknowledgments}

The authors thank the Particle Analysis Center of SFB 1214 at the University of Konstanz for measurements. Z.C. was funded by a Chinese Scholarship Council stipend. The authors gratefully acknowledge the computing time granted by the John von Neumann Institute for Computing (NIC) and provided on supercomputers JURECA and JUWELS at Jülich Supercomputing Center (JSC). This work was financially supported by the Sino-German Center for Research Promotion (Grants GZ 1351), the Deutsche Forschungsgemeinschaft (DFG) within the framework of the collaborative research center SFB 1214 projects A4 and B1 and the National Natural Science Foundation of China (21775142).

\section{Author contributions}

Z.C., M.H. and H.C. conceived the concept, designed the experiments, and wrote the manuscript. Z.C. synthesized and characterized the sample. Z.C. and X.W. performed data analysis. R.S. and P.N. developed the theoretical simulation part, performed theoretical simulation and data analysis and wrote the manuscript. M.F. and E.S. contributed to the characterization. Z.H. contributed to the calculation of surface energy.

\section{Competing interests}

The authors declare no competing financial interests.

\section{Additional information}

Supplementary Information is available for this paper.

Reprints and permissions information is available at www.nature.com/reprints. Correspondence and requests for materials should be addressed to M.H. or P.N. or H.C. 


\section{Supplementary Files}

This is a list of supplementary files associated with this preprint. Click to download.

- flatColfenspc.pdf

- 3SIColfen.docx 